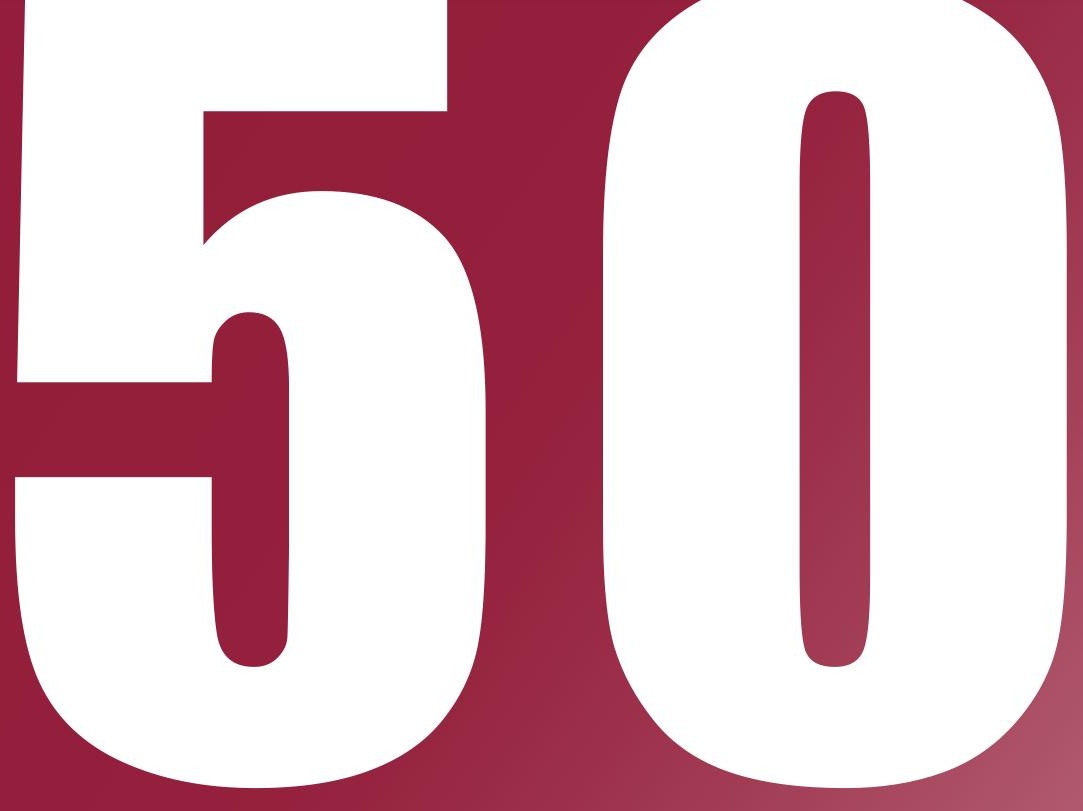

ÁMBITOS

REVISTA

INTERNACIONAL

DE

COMUNICACIÓN

$\mathbf{N}^{\circ} \mathbf{5 0}$

EDICIÓN OTOÑO

2020

ISSN: 1139-1979

E-ISSN: 1988-5733

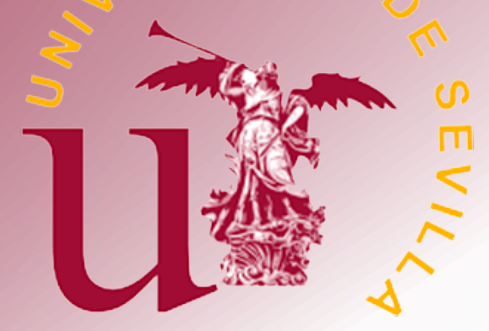




\section{INDICE}

EDITORIAL EDITORIAL

Presentación del Monográfico. Comunicación y juventud. Nuevos medios, representación, recepción y participación en contenidos de entretenimiento e información

Presentation of the monograph. Communication and youth. New media, representation, reception and participation in entertainment and information content.

Juan Francisco Gutiérrez Lozano, Francisco Javier Ruiz del Olmo

7-9

\section{MONOGRÁFICO MONOGRAPH}

El consumo audiovisual de la Generación Z. El predominio del vídeo online sobre la televisión tradicional The audiovisual consumption of Generation $Z$. The predominance of online video over traditional television

La imagen de los jóvenes en las estrategias transmedia de las series de televisión: el caso de Skam España en Instagram

The image of young people in transmedia strategies of TV series: the case of Skam España on Instagram

Sergio Jesús Villén Higueras, Francisco Javier Ruiz del Olmo

La penetración de Netflix en el público español ¿cuestiona el modelo televisivo tradicional?

Does the arrival of Netflix in the Spanish audience challenge the traditional television model?

Javier Bustos Díaz

49-61

Generación Z y consumo de información política: entre la televisión y los nuevos formatos mediáticos Generation $Z$ and consumption of political information: between television and new media formats

Ruth Gómez de Travesedo Rojas, Marta Gil Ramírez

$62-79$

Actualidad y comedia: El éxito de El Intermedio y La Resistencia entre los espectadores jóvenes

Information and comedy: The Success of El Intermedio and La Resistencia for young audiences

Inmaculada Concepción Aguilera García

80-95 
El efecto socializador de articular un espacio de comunidad virtual en el ecosistema del aula

The socializing effect of creating a virtual community space in the classroom environment

Lorea Ariadna Ruiz Gómez, Mónica Hinojosa Becerra, Francisco Javier Ruiz San Miguel

Juventude ciberativista e educação: reflexões sobre um jeito hacker de ser

Cyber activity youth and Education: reflections on a hacker way of being

Carla Azevedo de Aragão, Pietro Matheus Bompet Fontoura Alves, Karina Moreira Menezes

La risa grotesca en la obra de Bob Dylan: análisis de la Bacanal y el Festín Carnavalesco en la trilogía folk-rock eléctrica

Grotesque Laughter in Bob Dylan's Work: Analysis of The Bacchanal and the Carnival Feast in the Electric Folk-rock Trilogy

Jesús Albarrán Ligero

El papel del intertexto en el videojuego. Una partida, mil y una historias

Intertext in video games. A game, thousand and one stories

Rocío Serna-Rodrigo

145-158

\section{ÁmBitos PERSONALES PERSONAL ÁmBITOS}

El auge de Twitch: nuevas ofertas audiovisuales y cambios del consumo televisivo entre la audiencia juvenil The rise of Twitch: New audiovisual offers and the transformation of Television consumption among young audiences

\section{ARTÍCULOS ARTICLES}

La recepción de los medios de comunicación para residentes rusos en la Costa del Sol

Mass media reception by Russian residents on the Costa del Sol

Natalia Meléndez Malavé, José Carlos Pozo García

176-192

La calidad periodística en la cobertura de terremotos: Caso Ecuador

Journalistic quality in earthquake coverage: The case of Ecuador

Juan Pablo Arrobo-Agila, Mendoza María, Ignacio Aguaded

La credibilidad publicitaria en la nueva esfera mediática: los universitarios y los medios

Advertising credibility in the new media sphere: university students and the media 
Arco iris en medios brasileños: percepciones sociales sobre una campaña publicitaria LGBTQIA+

Rainbow in Brazilian media: social perceptions of an LGBTQIA+ advertising campaign

Leonardo Ferreira Batista, José Geraldo de Araújo Ramalho Filho

224-239

Rituales y comunicación política: la toma de posesión de los presidentes autonómicos españoles

Rituals and political communication: the Spanish autonomous Presidents inauguration

Ricardo Domínguez García

240-258

RESEÑAS REVIEWS

Una mirada diferente para hacer periodismo

A different look for making journalism

Guillermo Paredes-Otero

259-261

Periodismo y tecnología: una simbiosis continua

Journalism and technology: a continuous symbiosis

Luisa Graciela Aramburú Moncada

262-265

Una propuesta metodológica para impartir la asignatura de redacción periodística

Journalism and technology: a continuous symbiosis

Noelia Ruiz-Alba

266-268 


\title{
El consumo audiovisual de la Generación Z. El predominio del vídeo online sobre la televisión tradicional ${ }^{1}$
}

\author{
The audiovisual consumption of Generation Z. The predominance of online \\ video over traditional television
}

\author{
María Navarro Robles \\ Universidad CEU San Pablo | Paseo de Juan XXIII, no 10, 28040 Madrid | España \\ | https://orcid.org/0000-0002-7813-539X| maria.navarrorobles@ceu.es

\section{Tamara Vázquez Barrio} \\ Universidad CEU San Pablo | Paseo de Juan XXIII, no 10, 28040 Madrid | España \\ | https://orcid.org/0000-0003-2789-8554| tamarav@ceu.es
}

Fechas | Recepción: 31/05/2020 | Aceptación: 21/09/2020 | Publicación final: 15/10/2020

\section{Resumen}

La forma en la que consumimos contenido audiovisual ha cambiado. Las plataformas digitales y las redes sociales alojan contenidos que anteriormente solo podían encontrarse en la televisión. En consecuencia, cada vez son más los individuos que prefieren ver vídeos online a través de dispositivos electrónicos, especialmente entre las audiencias jóvenes. El objetivo de este trabajo es analizar el consumo audiovisual en la red de la Generación Z, jóvenes de 16 a 24 años, en comparación con el total de la audiencia. La metodología utilizada es una encuesta representativa a individuos con edades comprendidas entre los 13 y los 64 años residentes en la Comunidad de Madrid, aplicada cara a cara en el hogar del entrevistado y con una duración aproximada de 20 minutos. La muestra es de 1851 entrevistas, con un margen de error fijado en el $\pm 2,32 \%$ para datos globales, con variabilidad $p=q=0,5$ y un nivel de confianza del $95 \%$. Los resultados obtenidos confirman

\section{Abstract}

The way we consume audiovisual content has changed. Digital platforms and social networks host content that previously could only be found on television. Consequently, more and more individuals prefer to watch videos online through electronic devices, especially among young audiences. The objective of this communication is to analyze online audiovisual consumption in the Generation Z, young people between 16 and 24 years old, compared to the total audience. The methodology used is a representative survey of individuals aged between of 13 and 64 years residing in the Community of Madrid, applied face-to-face in the interviewee's home and lasting approximately 20 minutes. The sample is 1851 interviews, with a margin of error set at $\pm 2.32 \%$ for global data, with variability $p=q=0,5$ and a confidence level of $95 \%$. The results obtained confirm that young people are the users who best value audiovisual content online due to reasons such as spatiotemporal instantaneousness, the

Forma de citar:

Navarro Robles, M.; Vázquez Barrio, T. (2020). El consumo audiovisual de la Generación Z. El predominio del vídeo online sobre la televisión tradicional. Ámbitos. Revista Internacional de Comunicación 51, pp. 10-30. doi:

10.12795/Ambitos.2020.i50.02 
que los jóvenes son los usuarios que mejor valoran los contenidos audiovisuales online debido a motivos como la instantaneidad espaciotemporal, la variedad de contenidos o a la identificación con los creadores de los vídeos. De manera general, se concluye que los usuarios jóvenes, a diferencia de los adultos, se alejan de la televisión lineal concentrando su consumo en plataformas y soportes digitales. Resulta de especial interés analizar las razones del cambio, así como las elecciones de esta franja de audiencia a la hora de consumir contenido audiovisual.

Palabras clave: estudio de audiencia, televisión, medios electrónicos, red social, usuario de la comunicación. variety of content or the identification with the video creators. In general, it is concluded that young users, unlike adults, move away from traditional television concentrating their consumption on platforms and digital supports. It is of special interest to analyze the reasons for the change, as well as the choices of this audience segment when they consume audiovisual content.

\section{INTRODUCCIÓN}

La convergencia digital incide sobre los hábitos y conductas de los usuarios de medios. El desarrollo de Internet ha cambiado la forma en la que consumimos contenido audiovisual, ofreciendo productos que antes solamente podían encontrarse en la televisión.

El concepto de 'convergencia', según Jenkins (2008), se refiere al 'flujo de contenido a través de múltiples plataformas mediáticas, la cooperación entre múltiples industrias mediáticas y el comportamiento migratorio de las audiencias mediáticas, dispuestas a ir casi a cualquier parte en busca del tipo deseado de experiencias de entretenimiento" (p. 14-15).

Esta convergencia ha provocado cambios tanto a la hora de producir como de distribuir y promocionar los contenidos audiovisuales, unidos a una considerable modificación de los hábitos de consumo de las audiencias (Francisco-Lens \& Rodríguez-Vázquez, 2020, p. 208). De esta forma, el consumo televisivo se ha visto amenazado por la visualización de vídeos a través de Internet, que ofrece una mayor variedad de herramientas al consumidor (Hernández \& Martínez, 2016, p. 204).

Según el informe de Barlovento Comunicación (2019, p. 4-5), en agosto de 2019 el tiempo de navegación por Internet fue de 2 horas y 56 minutos diarios, mientras que los espectadores emplearon 5 horas y 17 minutos al día en ver la televisión. Con 21,4 millones de visitantes únicos, Internet consiguió una cobertura del $81,8 \%$ de la población mayor de edad. La televisión, por su parte, tuvo una audiencia acumulada de 35,1 millones, alcanzando al 91,2 \% de la población.

En el estudio realizado por Barlovento Comunicación (2020, p. 4-5) en abril de 2020, se observa que en este periodo los datos de consumo audiovisual aumentaron. El tiempo promedio de navegación en Internet por persona fue de 3 horas y 14 minutos diarios. Por otra parte, cada individuo pasó una media de 5 horas y 28 minutos viendo la televisión. Como se puede ver en el informe mencionado, en el mes de abril Internet logró 31,7 millones de visitantes únicos, suponiendo el $82,3 \%$ de la población. Sin embargo, la televisión continuó alcanzando cifras 
superiores, obteniendo una audiencia acumulada en ese mes de 35,4 millones, lo que se traduce en una cobertura del $91,7 \%$.

En el informe de IAB (2019, p. 38) se refleja que el 49,4\% de los individuos ven la televisión "menos que antes", el 35,2\% la consume "lo mismo que antes" y el 15,4\% lo hace "más que antes". Destaca una tendencia: el consumo de contenido audiovisual está comenzando a desplazar a los contenidos de la televisión tradicional en la audiencia menor de 45 años.

Como se puede observar, el consumo de televisión lineal todavía alcanza cifras superiores a las obtenidas por los contenidos audiovisuales online. Sin embargo, también se evidencia un aumento del consumo de vídeos en Internet, sobre todo entre las audiencias más jóvenes.

Según la Asociación para la Investigación de Medios de Comunicación (2020, p. 95), la visualización online de vídeos es la segunda actividad más realizada en Internet. Así mismo, el uso de plataformas tipo Netflix para ver películas o series destaca como una de las actividades preferidas por los usuarios.

Para Roel (2018), estas nuevas modalidades a la hora de visualizar contenido audiovisual se sitúan como una tendencia que se expande de forma progresiva y que, según la autora, "acabarán siendo -en un futuro no muy lejano- la opción prioritaria de consumo de contenidos audiovisuales" (p. 481).

Esta idea es compartida por Vázquez (2017, p. 35), quien asegura que la televisión tradicional no se encuentra preparada para luchar contra los soportes digitales, que gracias a Internet permiten ofrecer alternativas en las que los límites de la televisión desaparecen. De hecho, se están generando contenidos audiovisuales que se encuentran adaptados a la red, de forma que solo pueden ser consumidos a través de medios digitales (Casanova, 2011, p. 125). En consecuencia, las plataformas como Netflix logran ser cada vez más relevantes para el consumo audiovisual (Góngora \& Lavilla, 2020, p. 93).

Es indiscutible que los hábitos de consumo audiovisual están cambiando y la visualización de contenido en la red gana más peso a medida que pasa el tiempo. Pero a pesar de ello, la televisión continúa siendo el soporte preferido por una gran parte de la audiencia.

Así mismo, según Ruiz, Cáceres y Brändle (2010), aunque los jóvenes utilicen la red en gran medida para consumir contenido audiovisual, "también es cierto que el uso masivo de Internet no significa que los jóvenes hayan dejado de lado los contenidos televisivos tradicionales" ( $p$. 211).

En base a estos datos, el objetivo de nuestra investigación es conocer los hábitos de consumo audiovisual de los jóvenes tanto respecto a la televisión como respecto a los contenidos online. De esta forma, analizaremos el tiempo de visualización en cada uno de estos medios, cuáles son sus preferencias y qué soportes utilizan (Smart TV, plataformas digitales, etc.). A través de este estudio, trataremos de determinar las tendencias y los puntos de continuidad y discontinuidad entre la anterior sociedad de masas y la actual sociedad digital. 


\section{GENERACIÓN Z}

La Generación Z comprende a los jóvenes nacidos entre 1996 y 2003. ¿Quiénes son estos jóvenes y por qué nos centramos en ellos en este estudio? Según Álvarez, Heredia y Romero (2019, p. 2), sus principales características son las siguientes:

- Poseen un continuo deseo por estar en contacto e interaccionar con los demás, por lo que dan respuestas de forma inmediata.

- Son muy competentes con las tecnologías de la información (TIC), de las que han sido totalmente autodidactas.

- Tienen una gran facilidad para manejarse dentro del entorno digital, derivado de lo cual presentan preferencia por lo visual.

Son considerados nativos digitales y están hiperconectados, puesto que pasan sus días inmersos en las redes sociales realizando diversas actividades, entre las que destacan el ocio y las conversaciones con amigos. Según el Instituto Nacional de Estadística (2019), los individuos de esta franja de edad son los que hacen más uso de todos los servicios de Internet, contando la mayoría de ellos con habilidades digitales avanzadas. Son multitarea, multipantalla, muy creativos y tienen una mayor capacidad para la organización social (Aguilar, 2017, p. 4-16).

Al igual que los millennials, son una generación muy observada y analizada, sobre la que se conocen sus intereses, sus gustos e incluso sus comportamientos. Lo que une a todos los miembros de esta generación no es solo la edad, sino más bien el haber nacido a la misma vez que Internet. Esto ha provocado que su forma de relacionarse sea totalmente distinta a la de anteriores generaciones (Cerezo, 2016, p. 98).

Según Cerezo (2016, p. 105-106), la Generación Z se decanta por aplicaciones donde los contenidos desaparecen con rapidez, sin dejar ningún rastro. Además, la información de la que disponen en redes sociales está fragmentada y desestructurada, a diferencia de los medios tradicionales donde se encontraba jerarquizada.

Para ellos, el conocimiento es plano y no debe estar compuesto por jerarquías ni tampoco por compartimentos. De hecho, estos jóvenes desconocen los filtros que la información poseía en los medios tradicionales, puesto que ellos han crecido con el derecho de poder no solo crear, sino también participar en la información y transmitirla (Ortega, Soto \& Cerdán, 2016, p. 7-11).

Los jóvenes de 17 a 24 años aseguran que consumen muy poco los medios de comunicación tradicionales (o que incluso no los consumen). Esta generación se informa de manera distinta a las anteriores, poniendo el foco en el contenido presente en Internet, y no está dispuesta a "ceder ante el monopolio de los medios tradicionales" (Mouret, 2016, p. 167). En cuanto a los productos audiovisuales, su uso y consumo se inclina hacia canales interactivos a los que acceden a través de pantallas individuales y personalizadas (Ortega, González \& Pérez, 2015, p. 643).

Según Aranda, Roca y Sánchez (2013, p. 16), el 65 \% de los jóvenes escogería la red por encima de la televisión. De hecho, en Netflix y Youtube pasan el 59 \% de su tiempo, frente al 29 \% que 
están viendo televisión tradicional. (Trifecta Research, 2015, p. 7). Además, defienden la posibilidad de informarse a partir de diversas fuentes, que además sean gratuitas y libres, e invitan a los medios tradicionales a ofrecer una mayor participación y a ser más objetivos (Mouret, 2016, p. 107). Estas afirmaciones son apoyadas por Espiritusanto (2016): "esta desjerarquización, la crisis de credibilidad, los temas y las nuevas narrativas en las que se mueve la Generación $Z$ hacen que se comuniquen entre ellos como generadores y consumidores de contenido sin necesidad de grandes mediadores" (p. 125).

Respecto a los soportes, el 95 \% de los jóvenes tiene algún dispositivo móvil. Es muy llamativo el gran número de horas que pasan en el móvil: el $26 \%$ lo usa 10 horas o más al día. Esto conlleva que más de la mitad, concretamente el $58 \%$, afirmen que se sienten incómodos si no tienen sus teléfonos al menos durante unas horas. $Y$, de hecho, el $31 \%$ de ellos asegura sentir dicha incomodidad con tan solo estar lejos del móvil durante 30 minutos (The Center for Generational Kinetics, 2018, p. 6-8). Según Ozkan y Solmaz (2015, p. 96), el smartphone se sitúa como uno de los elementos más importantes de sus vidas.

Y es que la Generación $Z$ accede al mundo digital principalmente mediante el móvil, a través del cual establecen su relación con todo su entorno, desde amigos a profesores, puesto que han crecido en un mundo hiperconectado. Es por ello por lo que este grupo de edad ha provocado que el móvil deje de verse como un mero dispositivo para realizar llamadas, sino que pasa a ser un elemento integrador (Cerezo, 2016, p. 102).

Según Cerezo (2016), "si los baby boomers prefieren la comunicación cara a cara, la generación $X$ elige hablar por teléfono, aunque también usa el correo electrónico, mientras que la generación del milenio se dirige hacia las redes de medios sociales" (p. 104), lo cual es totalmente extensible a la Generación Z. Según las aportaciones de los jóvenes pertenecientes a la Generación Z presentes en Mouret (2016, p. 170), estos usuarios se reinventan cada día y se adaptan a los nuevos avances tecnológicos que surgen, los cuales ellos mismos promueven. Este cambio continuo justifica la oportunidad de esta investigación.

\section{METOdOlogía}

El objetivo de la presente investigación es analizar cómo influyen los usos de las redes sociales de la Generación $Z$ en el consumo audiovisual y compararlo con el del total de la audiencia. Este objetivo general se concreta en los siguientes objetivos específicos:

1. Analizar el consumo audiovisual de los jóvenes en televisión y a través de otros dispositivos. Identificar la frecuencia con la que los espectadores consumen contenido audiovisual y analizar a través de qué dispositivos lo hacen diferenciando especialmente entre la televisión tradicional y dispositivos tecnológicos digitales.

2. Mostrar la opinión de la audiencia sobre los programas de televisión y sobre los vídeos en línea e identificar los motivos de esas preferencias. 
En este estudio se utiliza una metodología de carácter cuantitativo a partir del diseño, aplicación y análisis de una encuesta representativa a individuos con edades comprendidas entre los 13 y los 64 años residentes en la Comunidad de Madrid.

El muestreo utilizado fue bietápico, estratificado por conglomerados, con selección de las unidades primarias de muestreo (municipios y secciones censales) de forma aleatoria proporcional y de las unidades últimas (individuos) por rutas aleatorias y cuotas de sexo y edad. La muestra es de 1851 entrevistas, con un margen de error fijado en el $\pm 2,32 \%$ para datos globales, con variabilidad $p=q=0,5$ y un nivel de confianza del $95 \%$. Los jóvenes de 17 a 24 años representan un $28,8 \%$ de la muestra total. En cuanto al resto de los grupos de edad los datos son los siguientes: los individuos entre 25 y 30 años suponen el $21,3 \%$ del total de la muestra; los adultos de 36 a 45 años son el 17,9\% del total; los de 45 a 55 años son el 17,7 \% y finalmente las personas de entre 56 a 65 años suponen el 14,3\% de la muestra. En cuanto al sexo, el 49,2 $\%$ de los encuestados son hombres y el $50,8 \%$ son mujeres.

La encuesta fue aplicada cara a cara en el hogar del entrevistado mediante el sistema CAPI con una duración de 20 minutos. El cuestionario se estructuró en tres grandes bloques: preguntas sobre el consumo de televisión, preguntas sobre el consumo concurrente y preguntas sobre el consumo audiovisual a través de dispositivos móviles. Esta investigación se centra en el primero $\mathrm{y}$ tercero de estos bloques.

Antes de comenzar la fase de campo, se realizó un pretest de 15 entrevistas personales en el hogar de los entrevistados para corregir posibles errores (falta de comprensión de preguntas, filtros de las mismas, orden de las preguntas, etc.), con el fin de subsanarlos en el cuestionario final. El trabajo de campo fue realizado por la empresa Idea Solutions entre el 17 de junio y el 4 de julio de 2019.

A partir de los datos recabados con la aplicación de la encuesta, se realiza un análisis descriptivo sobre el consumo audiovisual de los jóvenes españoles en comparación con el resto de la población.

\section{RESULTADOS}

Los resultados obtenidos a través de la encuesta se van a presentar divididos en tres bloques. EI primero de ellos está destinado a mostrar cómo es el consumo audiovisual de la audiencia en los diferentes soportes disponibles. Posteriormente, analizamos de forma concreta su consumo de televisión. $Y$, finalmente, procedemos a describir el consumo audiovisual en línea de los usuarios.

\subsection{Consumo audiovisual en los diferentes soportes}

En primer lugar, vamos a analizar qué soportes elige la audiencia para consumir contenido audiovisual. Según nuestra encuesta, los individuos visualizan contenidos audiovisuales entre 2 y 4 horas al día, aumentando esta cifra en los días festivos y los fines de semana. Las clases más altas son quienes consumen con más frecuencia este contenido en todos los dispositivos, 
excepto en la televisión. En el caso de este medio, son las clases más bajas quienes lo utilizan más significativamente.

El smartphone y la televisión se posicionan como los soportes en los que más se consume contenido audiovisual. Se trata de un hecho que se da tanto en los días de diario como en los fines de semana. En concreto, el 22,6\% de los encuestados utiliza el smartphone para ver vídeos durante más de 5 horas en días de diario y el 21,0\% hace lo mismo en fines de semana y festivos. Por su parte, la televisión tradicional es visualizada entre 1 y 2 horas por el 32,0 \% de la audiencia en los días de diario y entre 2 y 3 horas por el $26,4 \%$ de los individuos en los fines de semana.

El 25,0 \% del total de la población visualiza contenido audiovisual en la televisión durante 2 o 3 horas en los días de diario, mientras que el $15,6 \%$ lo hace en el smartphone. El siguiente dispositivo más utilizado es el ordenador (11,6\%) y en último lugar se encuentra la tablet (6,2\%).

Como indicábamos anteriormente, todas estas cifras aumentan en los fines de semana, cuando el 44,3\% de los encuestados consume contenido audiovisual en televisión durante un tiempo medio de entre 2 y 4 horas. El 23,1 \% lo hace en smartphone, el 15,9\% en ordenador y el 9,9\% en tablet.

\begin{tabular}{|c|c|c|c|c|c|}
\hline 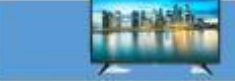 & $\begin{array}{l}\text { Dias de } \\
\text { diario }\end{array}$ & $\begin{array}{l}\text { Fines de } \\
\text { semana }\end{array}$ & & $\begin{array}{l}\text { Dias de } \\
\text { diario }\end{array}$ & $\begin{array}{l}\text { Fines de } \\
\text { semana }\end{array}$ \\
\hline Low viewers & $45,0 \%$ & $28,4 \%$ & Low viewers & $37,1 \%$ & $32,0 \%$ \\
\hline Medium viewers & $25,0 \%$ & $44,3 \%$ & Medium viewers & $15,6 \%$ & $23,1 \%$ \\
\hline High viewers & $26,5 \%$ & $23,6 \%$ & High viewers & $33,6 \%$ & $30,0 \%$ \\
\hline 2 & $\begin{array}{l}\text { Dias de } \\
\text { diario }\end{array}$ & $\begin{array}{l}\text { Fines de } \\
\text { semana }\end{array}$ & & $\begin{array}{c}\text { Dias de } \\
\text { diario }\end{array}$ & $\begin{array}{l}\text { Fines de } \\
\text { semana }\end{array}$ \\
\hline Low viewers & $32,7 \%$ & $29,5 \%$ & Low viewers & $25,3 \%$ & $21,3 \%$ \\
\hline Medium viewers & $11,6 \%$ & $15,9 \%$ & Medium viewers & $6,2 \%$ & $9,9 \%$ \\
\hline High viewers & $12,2 \%$ & $8,6 \%$ & High viewers & $4,6 \%$ & $3,7 \%$ \\
\hline
\end{tabular}

Figura 1. Consumo audiovisual en distintos soportes

Fuente: elaboración propia.

Los usuarios jóvenes de entre 17 y 24 años, es decir, aquellos pertenecientes a la Generación Z, suelen visualizar el contenido audiovisual tanto en el ordenador como en la tablet y el móvil, situando estos dispositivos como preferidos frente a la televisión. Este dato es muy diferente en los grupos de audiencias más mayores, quienes consumen con más frecuencia y durante un mayor número de horas la televisión tradicional.

\subsection{Consumo de televisión}

Para analizar el consumo de televisión por parte de la audiencia, hemos preguntado a los encuestados acerca de los siguientes temas: el recuerdo televisivo de hace 5 años, la suscripción a plataformas audiovisuales de pago, la frecuencia de visualización de programas y cadenas en 
televisión, la valoración de la televisión y la preferencia por programas de televisión frente a vídeos online.

\subsubsection{Recuerdo televisivo de hace 5 años}

Aunque el 39,2 \% de los encuestados afirman que visualizan la misma televisión que hace 5 años, el 12,8 \% confirma que la ve menos porque dedica más tiempo a navegar por Internet y a redes sociales en general, mientras que el 6,7 \% reconoce verla menos por haberla sustituido por dispositivos móviles, donde ve series, películas y programas de televisión. Sin embargo, solo el $0,7 \%$ ha cambiado la televisión por los vídeos de youtubers, de instagramers o por vídeos de personas anónimas de Internet.

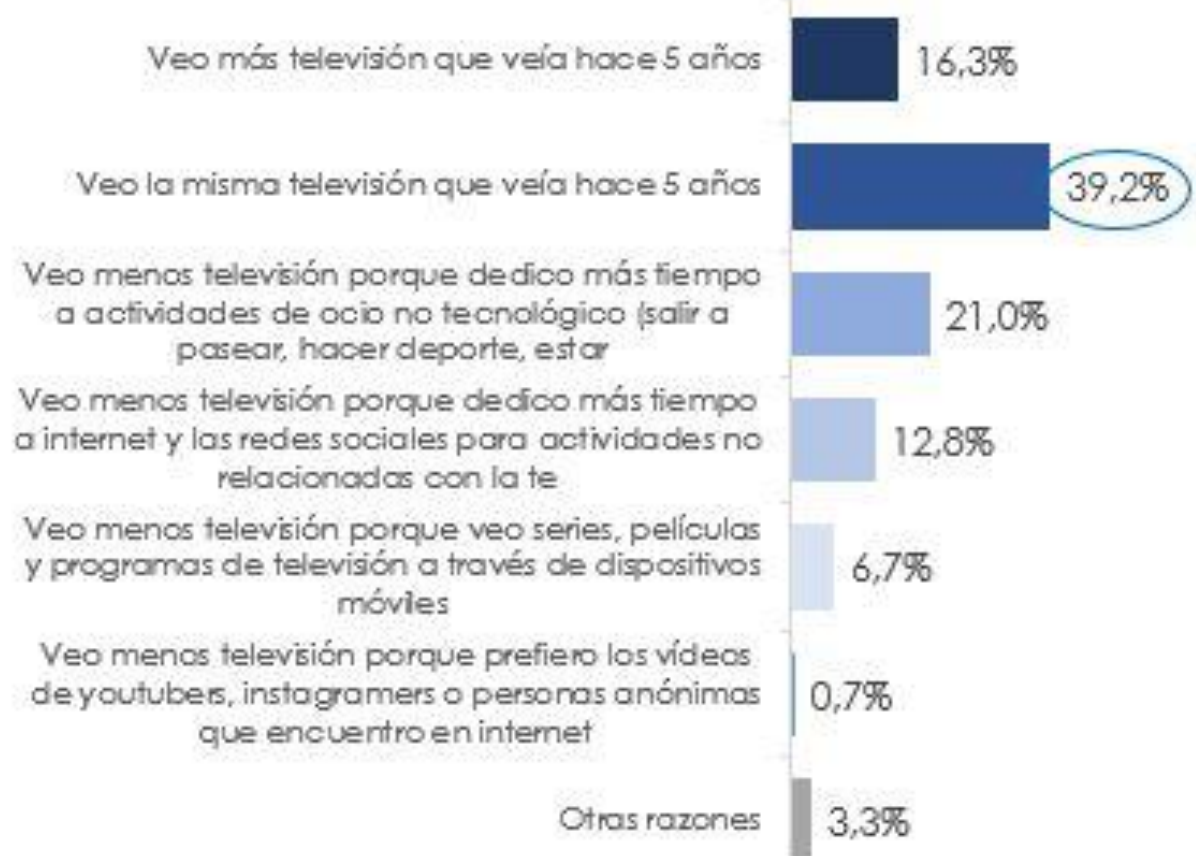

Figura 2. Recuerdo televisivo de hace 5 años

Fuente: elaboración propia.

\subsubsection{Suscripción a plataformas audiovisuales de pago}

Cuando preguntamos acerca de la suscripción a alguna plataforma de pago para ver contenidos audiovisuales, el 52,4 \% de los individuos contestó afirmativamente. Entre las plataformas planteadas, Netflix es la que reúne más audiencia, alcanzando el 69,1 \%. Le sigue Movistar, que cuenta con el $29,4 \%$ y HBO, con el $20,8 \%$. Las plataformas pertenecientes a las compañías telefónicas obtienen cifras más bajas: Vodafone posee el $10,7 \%$ de la población suscrita a alguna plataforma, mientras que Orange tiene el 10,5\%. En último lugar, encontramos Prime Vídeo (8,9 $\%)$ y Sky $(1,2 \%)$. 


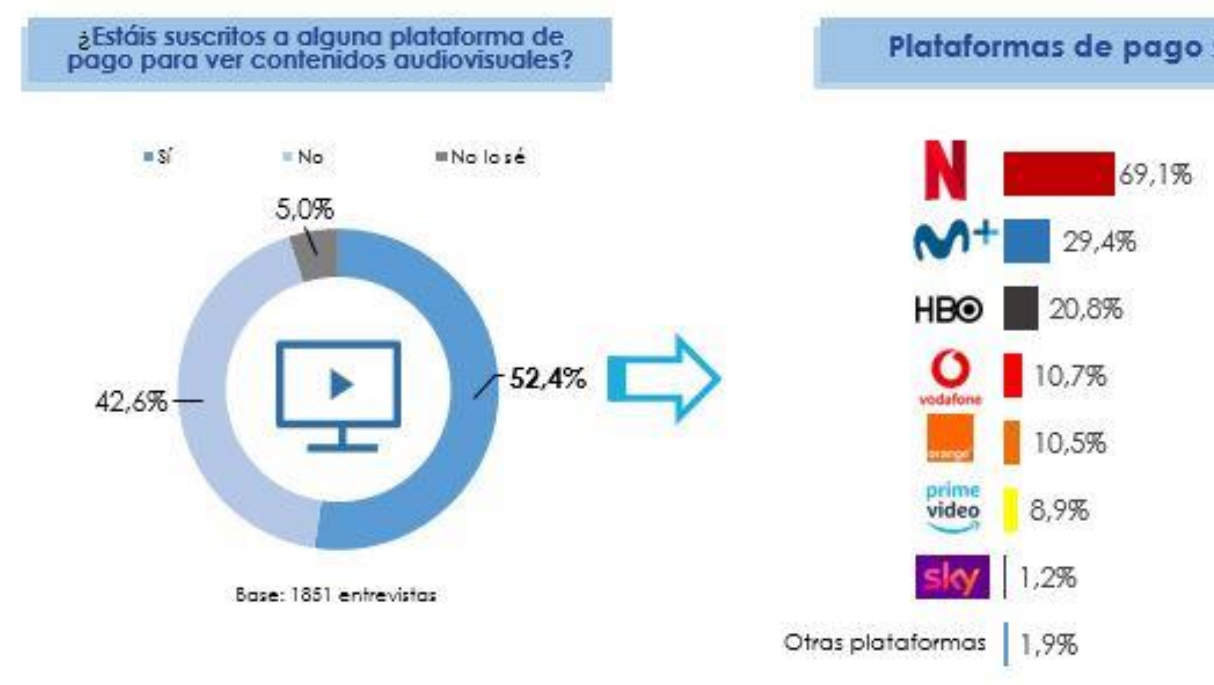

Figura 3. Suscripción a plataformas audiovisuales de pago Fuente: elaboración propia.

\subsubsection{Frecuencia de visualización de programas y cadenas en televisión}

Las películas y las series son los programas que la audiencia consume todos o casi todos los días en televisión, con un $45,8 \%$ y un $44,9 \%$ respectivamente. A su vez, los talent shows y los dating shows se posicionan como los menos visualizados: un 9,6 \% de los encuestados ve los talents diariamente y solo un $9,3 \%$ ve los dating shows con tal asiduidad. Sin embargo, estas cifras varían cuando diferenciamos entre las cadenas de televisión en abierto y las cadenas de pago tales como Movistar, Orange o Netflix. En este caso, los programas más visualizados en la televisión en abierto son los informativos $(85,5 \%)$, los realities shows $(84,7 \%$ ) y los talent shows $(84,1 \%)$. Al contrario de los resultados generales, entre los programas que menos ve la audiencia en las cadenas de televisión destacan las series $(65,6 \%)$ junto a las películas $(67,7 \%)$ y los eventos deportivos (67,7 \%). En las cadenas de pago, sin embargo, las películas es uno de los contenidos más demandados (58,6\%), además de las series de televisión (53,1\%). Los resultados más bajos corresponden a los concursos ( $8,8 \%$ y a los realities shows $(10,0 \%)$.

Un $88,7 \%$ de los encuestados afirma visualizar los programas informativos en directo, al igual que los talent shows (88,7\%) y los magazines y talk shows (88,3\%). Las series de televisión (68,9 $\%$ ) y las películas $(58,6 \%$ ) son los contenidos menos preferidos para ver en esta modalidad.

A su vez, de aquellos que acceden al contenido en diferido, un $53,8 \%$ se decantan por las películas y un $49,1 \%$ por las series de televisión. Solo un $7,9 \%$ ve los concursos de esta manera y un $9,5 \%$ de los espectadores elige esta opción para los magazines y los talk shows, que como hemos visto anteriormente suelen visualizarse en directo. 


\begin{tabular}{|c|c|c|c|c|c|}
\hline \multirow[t]{2}{*}{ Programas } & Frecuencia de ver TV & \multicolumn{2}{|c|}{ Donde ve los contenidos } & \multicolumn{2}{|c|}{$\begin{array}{c}\text { Como suele ver los } \\
\text { contenido }\end{array}$} \\
\hline & $\begin{array}{l}\text { Top } 2 \text { Box } \\
\text { (Todos o casi todos los días } \\
+3 / 4 \text { veces semana) }\end{array}$ & $\begin{array}{c}\text { Cadenas de TV en } \\
\text { abierto }\end{array}$ & $\begin{array}{l}\text { Cadenas de pago } \\
\text { (Movistar, Orange, Netflix...) }\end{array}$ & Directo & Diferido \\
\hline Películas & 4 & & & & \\
\hline Series de televisión & $\because 44,9 \%$ & & & & \\
\hline Programas informativos & $39,0 \%$ & & $14,4 \%$ & & $11,6 \%$ \\
\hline Programas del corazón & $18,6 \%$ & $80,3 \%$ & $12,2 \%$ & $85,1 \%$ & $12,9 \%$ \\
\hline Concursos & $16,4 \%$ & $82,3 \%$ & & $86,2 \%$ & \\
\hline Reality shows & $15,6 \%$ & $84,7 \%$ & $4.10,0 \%$ & $87,7 \%$ & $12,6 \%$ \\
\hline Eventos deportivos & $15,4 \%$ & 67.7 & $36,8 \%$ & $76,6 \%$ & $35,0 \%$ \\
\hline
\end{tabular}

Figura 4. Frecuencia de consumo audiovisual en televisión I

Fuente: elaboración propia.

\begin{tabular}{|c|c|c|c|c|c|}
\hline \multirow[t]{2}{*}{ Programas } & Frecuencia de ver TV & \multicolumn{2}{|c|}{ Donde ve los contenidos } & \multicolumn{2}{|c|}{$\begin{array}{c}\text { Como suele ver los } \\
\text { contenido }\end{array}$} \\
\hline & $\begin{array}{l}\text { Top } 2 \text { Box } \\
\text { (Todos o casi todos los días } \\
+3 / 4 \text { veces semana) }\end{array}$ & $\begin{array}{c}\text { Cadenas de TV en } \\
\text { abierto }\end{array}$ & $\begin{array}{l}\text { Cadenas de pago } \\
\text { (Movistar, Orange, Netflix...) }\end{array}$ & Directo & Diferido \\
\hline Películas & 070 & & & & \\
\hline Series de televisión & $\because .44,9 \%$ & & & & \\
\hline Programas informativos & $39,0 \%$ & 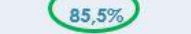 & $14,4 \%$ & & $11,6 \%$ \\
\hline Programas del corazón & $18,6 \%$ & $80,3 \%$ & $12,2 \%$ & $85,1 \%$ & $12,9 \%$ \\
\hline Concursos & $16,4 \%$ & $82,3 \%$ & & $86,2 \%$ & \\
\hline Reality shows & $15,6 \%$ & $84,7 \%$ & $10,0 \%$ & $87,7 \%$ & $12,6 \%$ \\
\hline Eventos deportivos & $15,4 \%$ & 67,7 & $36,8 \%$ & $76,6 \%$ & $35,0 \%$ \\
\hline
\end{tabular}

Figura 5. Frecuencia de consumo audiovisual en televisión II

Fuente: elaboración propia.

En el caso de los jóvenes, resulta llamativo que, aunque los realities shows son vistos por todos los grupos de edad, posicionándose como uno de los contenidos que más se consumen en la televisión, el grupo de entre 17 y 24 años es significativamente el que menos los ve.

En las plataformas de pago, los jóvenes sí que acceden para consumir un contenido coincidente con el elegido por las generaciones más mayores. Es el caso de los eventos deportivos, cuyos principales espectadores comprenden desde los 17 hasta los 45 años.

También sucede algo llamativo en el consumo de las películas: los grupos que significativamente ven más este contenido son los más mayores, de entre 56 y 65 años, y los más jóvenes, de entre 17 y 24 . Sin embargo, respecto a las series, el grupo más relevante de espectadores está formado por mujeres de entre 17 y 24 años. Cabe destacar que tanto las películas como las series y los documentales son mucho más vistos mediante las cadenas de pago como Netflix o HBO por la 
franja de edad de 25 a 35 años, aunque los usuarios de 17 a 24 años también las consumen en dichas plataformas con mucha frecuencia.

\subsubsection{Valoración de la televisión}

La opinión de la mayoría de los grupos de edad es que el mayor punto débil de la televisión es el exceso de anuncios: el 56,8 \% de los encuestados afirma que "en la televisión hay demasiada publicidad". Sin embargo, el 48,3 \% dice estar "bastante de acuerdo" en que este medio ofrece una gran variedad de programas para entretenerse. Otro factor positivo es que el 35,5 \% está "bastante de acuerdo" en que este medio le permite compartir el visionado con sus familiares y amigos. Sin embargo, el 40,1 \% asegura que los programas se emiten demasiado tarde.

Cuando se les pregunta por su preferencia por los vídeos online frente a los programas de televisión, las opiniones son muy variadas: el 28,4 \% dice estar "ni de acuerdo ni en desacuerdo", mientras que el $26,6 \%$ asegura estar "totalmente de acuerdo" con dicha preferencia. Lo mismo sucede con la elección de los dispositivos móviles frente a la televisión, con un $28,3 \%$ de los encuestados que no tienen ninguna opinión clara al respecto y un $24,6 \%$ que admite preferir dichos dispositivos.

Como cabía esperar, el grupo más mayor (de 56 a 65 años) es el que valora más positivamente la televisión. De manera general consideran que aporta variedad en su programación y afirman que supone un medio de sociabilización, motivos por los que la prefieren frente a los contenidos audiovisuales online.

Sin embargo, los grupos de edad media, como el de 35 a 45 años, ya comienzan a ver aspectos negativos en este soporte, afirmando que tiene demasiada publicidad, siendo esta la razón por la que deciden sustituirla por plataformas de pago. El grupo de edad más joven, de 17 a 24 años, considera que la televisión es un instrumento que resulta muy útil para informarse, pero que no les ofrece un tipo de programación que se adapte a su personalidad.

\subsubsection{Preferencia por programas de televisión frente a vídeos online}

Aunque un alto porcentaje de los encuestados, concretamente el $42,0 \%$, asegura que le gusta consumir vídeos online y programas de televisión por igual, existe un mayor número de personas que prefieren la televisión (42,9\%) frente a los vídeos online $(15,0 \%)$. 


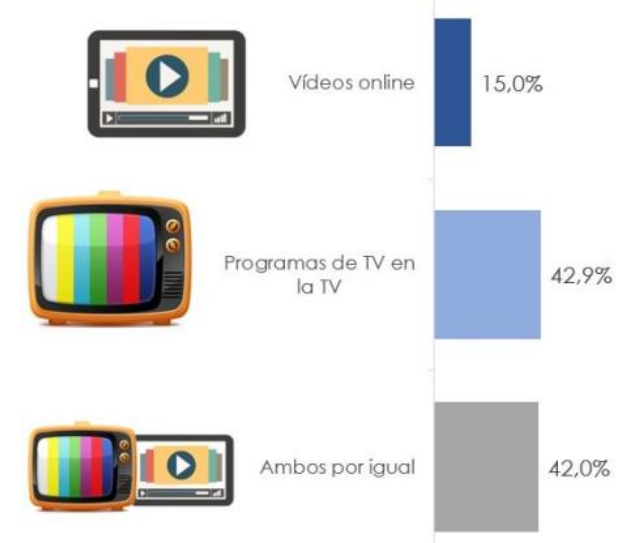

Figura 6. Preferencia por programas de televisión frente a vídeos online Fuente: elaboración propia.

Sin embargo, los datos varían mucho en función de la edad. Por una parte, los más mayores, concretamente adultos de entre 46 y 65 años, optan por los programas de televisión. Por otra parte, los jóvenes de entre 17 y 35 años prefieren los vídeos online más que cualquier otro grupo de edad. Cabe destacar que la mayoría de los encuestados que afirmaban no tener preferencia frente a ninguna de las dos opciones pertenecían al grupo de entre 17 y 24 años.

\subsection{Consumo de contenido audiovisual online}

Tras conocer cómo es el consumo televisivo de la audiencia, y con el fin de compararlo con su consumo online de contenido audiovisual, preguntamos a los encuestados acerca de los siguientes aspectos: la frecuencia de consumo de contenidos audiovisuales online por dispositivo, los contenidos más vistos por tipo de dispositivo, la preferencia por las diferentes plataformas online para ver programas de televisión y las motivaciones y el sentido de la utilización de la red para ver contenido audiovisual.

\subsubsection{Frecuencia de consumo de contenidos audiovisuales online por dispositivo}

Como ya se comentaba anteriormente, el dispositivo más utilizado para el visionado de contenido audiovisual online es el smartphone. De esta forma, el ordenador pierde el puesto como dispositivo más utilizado para ver vídeos en Internet. La razón de este cambio se debe a que en el propio teléfono móvil se integran los demás dispositivos, desde el ordenador portátil hasta la televisión.

En este caso, el 52,6 \% de los encuestados afirma no ver "nunca o casi nunca" la televisión. Esta cifra no dista mucho de la que obtienen la tablet $(67,4 \%)$ ni el ordenador $(48,2 \%)$. Sin embargo, existe una clara diferencia respecto al smartphone, el cual es utilizado a diario por el 37,9\% de los individuos y solo un $15,8 \%$ dice no usarlo "nunca o casi nunca". 

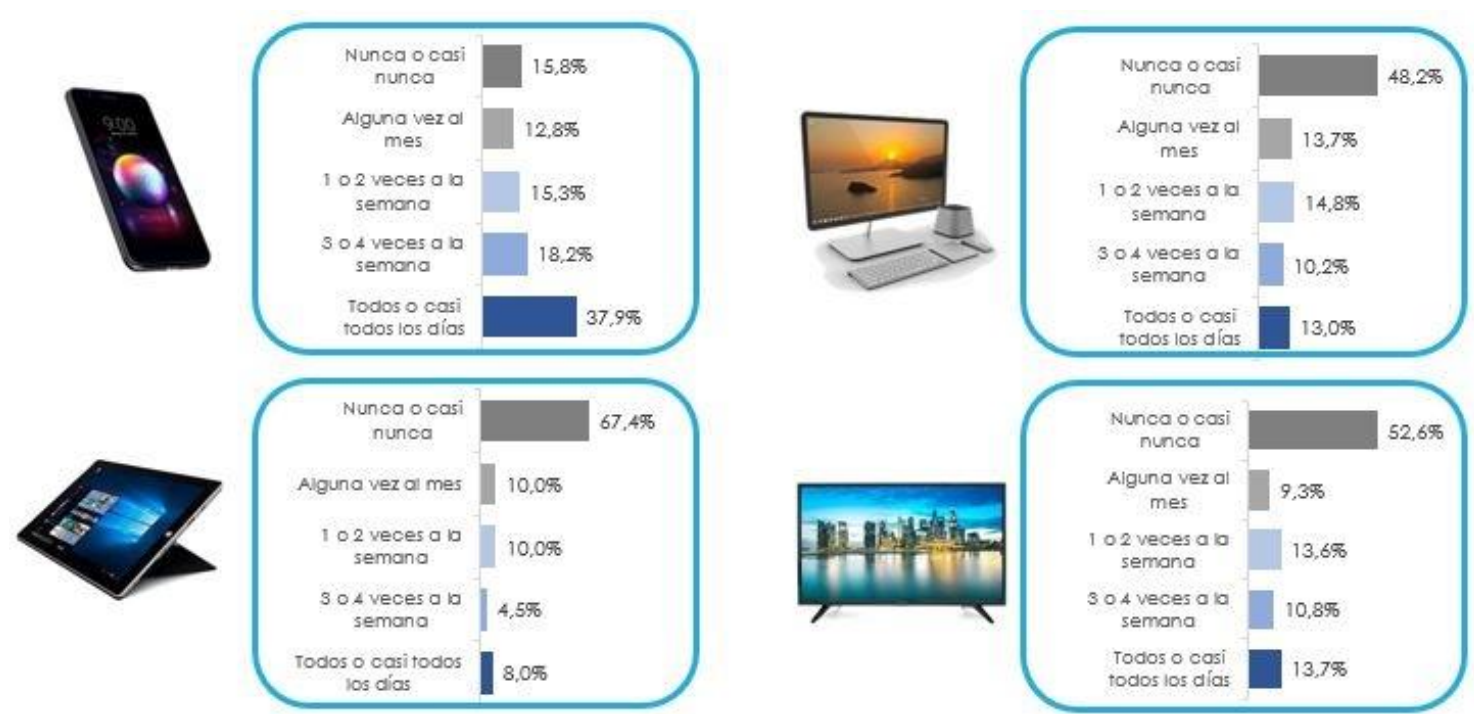

Figura 7. Frecuencia de consumo de contenidos audiovisuales online por dispositivo Fuente: elaboración propia.

Los jóvenes de entre 17 y 24 años son el grupo de edad que más ve contenido audiovisual en todos los dispositivos que analizamos en el estudio. Además, coincide que aquellas personas que obtienen datos más altos en todos los dispositivos son también quienes consumen más plataformas de pago.

\subsubsection{Contenidos más vistos por tipo de dispositivo}

Cuando preguntamos a los encuestados por el soporte que utilizaban preferentemente a la hora de visualizar contenido audiovisual, la mayoría de ellos optaban por el smartphone, seguido de la Smart TV, el ordenador y finalmente la tablet.

En concreto, los usuarios utilizan el smartphone para visualizar vídeos musicales (52,2\%), vídeos graciosos de bromas $(50,6 \%)$ y vídeos realizados por amigos $(45,9 \%)$. Sin embargo, prácticamente no acceden a través de este soporte a los programas de televisión $(11,1 \%)$ ni a los vídeos de gente jugando a videojuegos (13,2 \%). El cine y los deportes también obtienen cifras bajas en este soporte.

El ordenador, por su parte, es utilizado principalmente para ver series $(25,8 \%)$, cine $(22,4 \%)$ y vídeos musicales (21,7\%). Los datos más bajos para este dispositivo corresponden de nuevo a los programas de televisión (5,8 \%) y los vídeos domésticos de personas anónimas (5,9\%).

Los encuestados afirmaron utilizar la tablet esencialmente para las series $(18,4 \%)$, seguido de los vídeos musicales $(14,7 \%$ ) y el cine $(13,3 \%)$, aunque es muy poco empleada a la hora de ver programas de televisión $(3,5 \%$ y vídeos de jugadores de videojuegos $(3,7 \%)$.

La visualización de series también destaca en Smart TV (41,1\%), junto al cine (35,8 \%) y los programas de televisión (22,6\%). Así mismo, el acceso a vídeos de gente jugando a videojuegos y vídeos domésticos también es muy bajo en este soporte (3,7 \% y 4,5\%, respectivamente). 


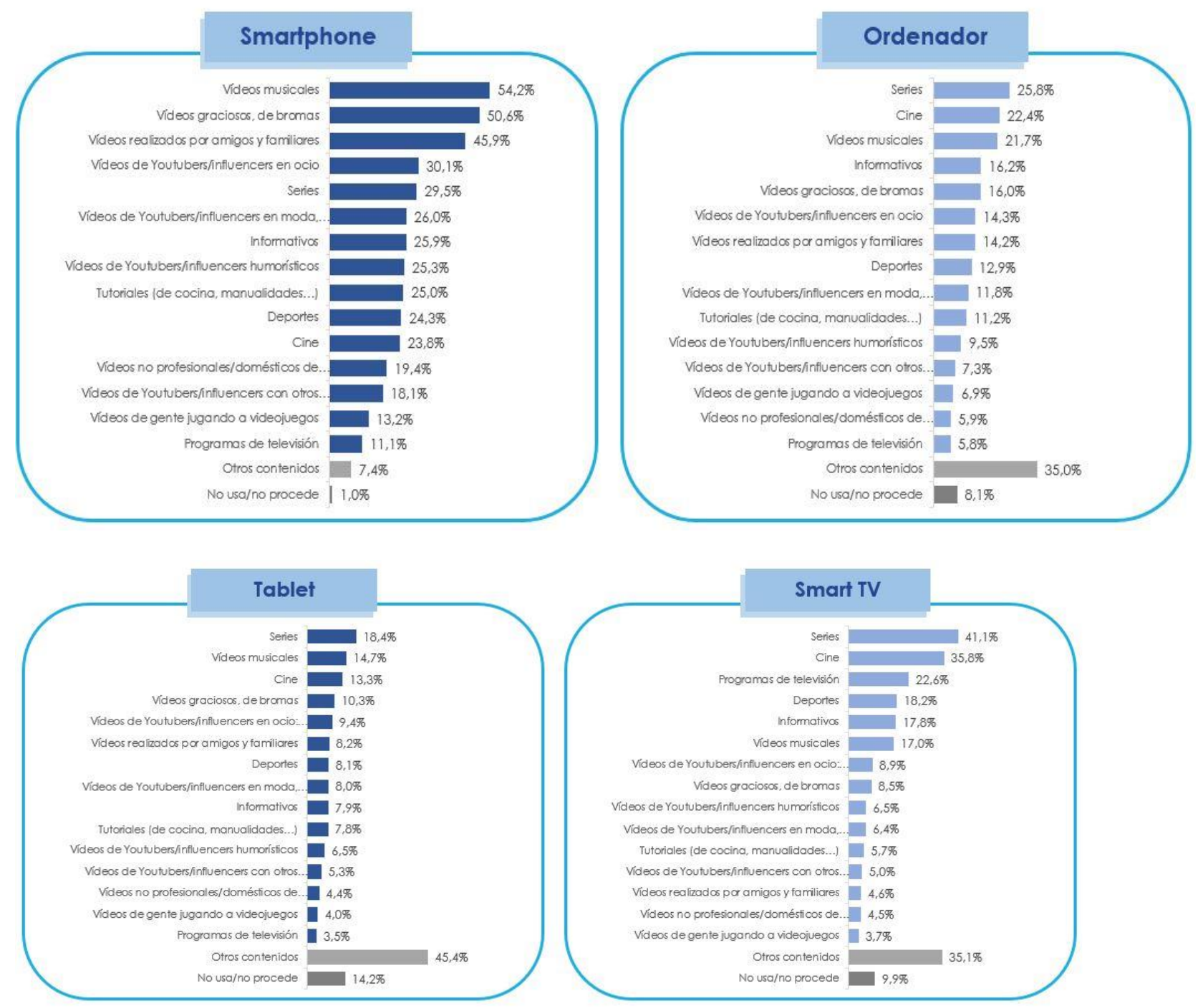

Figura 8. Soporte utilizado para ver contenido audiovisual

Fuente: elaboración propia.

Los jóvenes de entre 17 y 35 años son los que visualizan más contenidos audiovisuales en el teléfono móvil, obteniendo datos con significación en todas las categorías de respuesta. En cuanto al resto de dispositivos, puede observarse la misma tendencia: se trata del grupo que más ve contenidos audiovisuales en todos los soportes analizados.

Cabe destacar, igualmente, los altos porcentajes que obtiene la smart TV en este grupo, siendo el segundo dispositivo más utilizado para la visualización de series y películas. Además, aunque los jóvenes sean los que utilizan más este dispositivo para ver dichos contenidos, los perfiles de edad más altos también obtienen datos elevados, observándose cómo los más mayores empiezan a adentrarse en el mundo digital.

\subsubsection{Preferencia por las diferentes plataformas online para ver programas de televisión}

En general, las páginas web oficiales de las cadenas de televisión en abierto son las plataformas online más utilizadas para ver programas de televisión, siendo los informativos de noticias el programa más visualizado a través de este medio. 
Un 63,5\% de la audiencia accede a través de los portales web de las cadenas televisivas para ver los informativos. También son significativamente utilizados para ver los deportes $(54,6 \%)$, series y películas $(53,9 \%)$ e incluso programas infantiles $(47,6 \%)$.

Sin embargo, las aplicaciones de las cadenas, como por ejemplo Atresplayer o +TVE, resultan ser muy poco utilizadas: solo un 3,6 \% de la audiencia las usa para ver series y películas, un 3,1\% para ver deportes, un 1,9\% para ver informativos y solo un 1,3\% para programas infantiles.

Las plataformas de pago, tales como Netflix y HBO, y los servicios de streaming son utilizados por el $37,2 \%$ de los encuestados para ver series y películas, mientras que el $8,9 \%$ los utiliza para ver deportes, el 6,1 \% para programas infantiles y solo el 3,3 \% para ver los informativos.

Por su parte, las plataformas de las compañías telefónicas (Movistar, Vodafone, Orange, etc.) son usadas principalmente para las series y las películas $(24,1 \%)$, seguidas de los deportes $(16,4$ $\%)$, los informativos ( $8,3 \%$ ) y finalmente los programas infantiles $(8,0 \%)$.

Un $13,2 \%$ de la audiencia suele acudir a Youtube para ver series y películas y un 10,1\% ve los deportes en esta plataforma. Así mismo, un $6,4 \%$ de los encuestados la emplea para ver los informativos y un $5,5 \%$ para ver programas infantiles.

Las redes sociales son principalmente utilizadas, aunque de forma muy reducida, para ver informativos $(4,6 \%)$ y deportes $(4,4 \%)$. Y obtienen datos aún más bajos para las series y películas $(2,9 \%)$, que no suelen estar presentes en este tipo de web, y para los programas infantiles $(1,2$ $\%)$.

Más allá de la visualización online, la audiencia también descarga los contenidos audiovisuales de Internet. Esto sucede primordialmente en el caso de las películas y las series $(17,9 \%)$, aunque la cifra es mucho más baja cuando buscan consumir deportes $(5,3 \%)$, informativos $(3,7 \%)$ y programas infantiles $(2,6 \%)$.

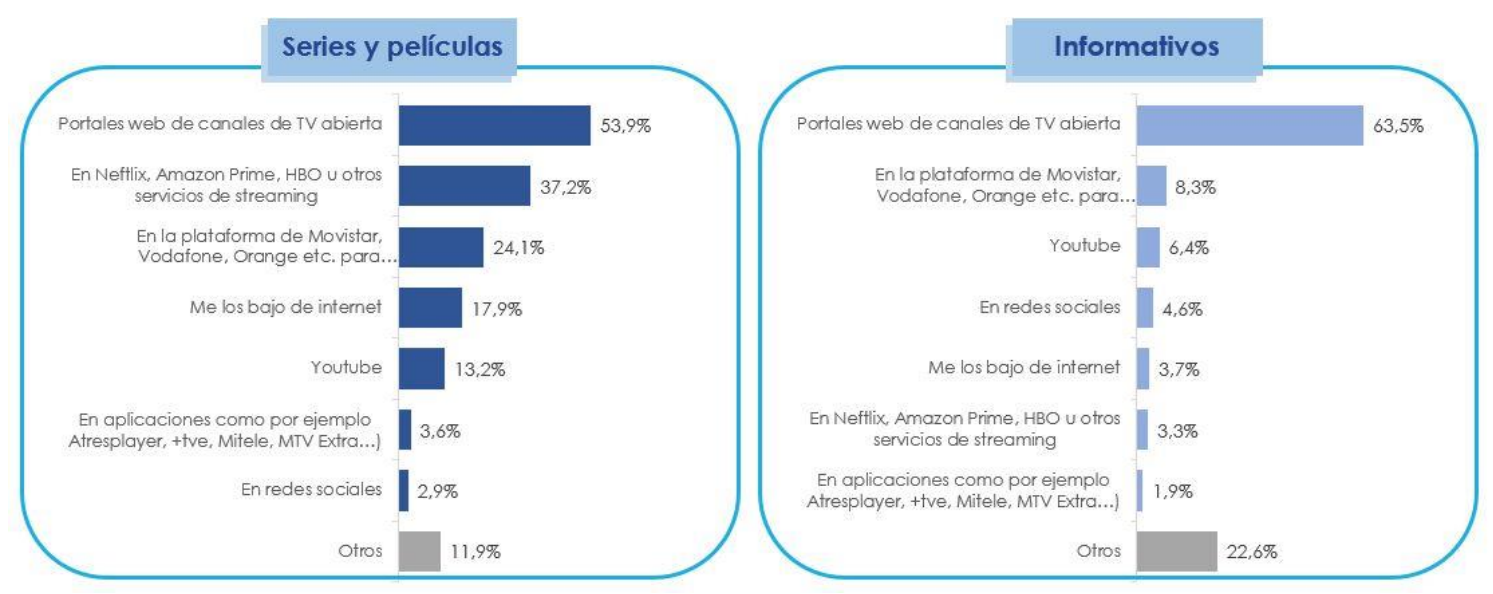



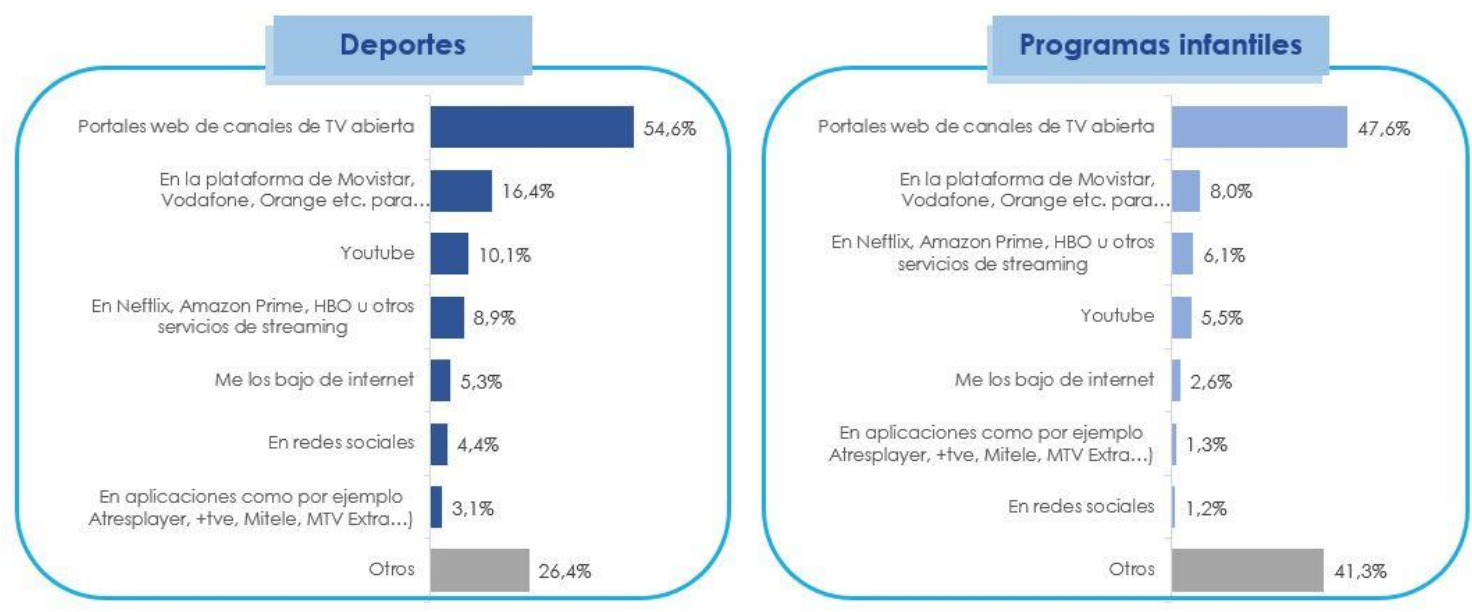

Figura 9. Preferencia por plataformas online

Fuente: elaboración propia.

La audiencia joven es la que hace uso de una mayor variedad de opciones para ver series y películas a través de Internet, observándose datos relevantes en diferentes categorías. Los jóvenes pertenecientes a la franja de edad de entre 17 y 24 años visualizan mucho más estos contenidos descargándoselos de Internet, así como a través de las plataformas de pago como Netflix y HBO e incluso a través de las propias redes sociales.

En relación con los programas, los informativos no son muy vistos entre los jóvenes, puesto que ni siquiera acceden a ellos a través de plataformas online. Sin embargo, cabe destacar que los grupos de edad de entre 17 y 35 años obtienen datos muy altos en la visualización de deportes a través de Netflix, HBO y otros servicios de streaming.

\subsubsection{Motivaciones y sentido de la utilización de la red para ver contenido audiovisual}

Según nuestra encuesta, se observa que lo más valorado de los contenidos audiovisuales online es su instantaneidad espaciotemporal, unida al hecho de no contar con publicidad. También destacan aspectos relacionados con el contenido intrínseco de los vídeos, como la variedad de contenidos, el interés hacia los mismos, la posibilidad de ver vídeos creados por youtubers o influencers famosos y que sus creadores sean personas parecidas a la audiencia.

Si nos fijamos en los datos obtenidos individualmente, encontramos que el $49,8 \%$ de los encuestados aseguran que es "muy importante" para ellos poder ver los contenidos cuándo y donde quiera, mientras que el 33,7 \% lo declara como "algo importante". De media, el 4,26 sobre 5 de los espectadores posiciona esta instantaneidad como el principal motivo para consumir contenido audiovisual en Internet.

Otro de los sentidos más relevantes de la utilización de la red para ver vídeos online es la posibilidad de evitar la publicidad de la televisión cuando se ven programas televisivos. En este caso, el 44,9 \% afirma que esta razón es "muy importante para ellos" y el 35,8 \% que es "algo importante". De esta forma, el 4,18 sobre 5 de los encuestados sitúa la ausencia de publicidad como principal factor de interés.

El tercer aspecto más considerado como más relevante para la audiencia es la variedad de contenidos: para el 44,2 \% es "muy importante" y para el 38,1\% resulta "algo importante". Este motivo logra alcanzar una media del 4,19 sobre 5 de espectadores. 
El hecho de mantenerse informado es considerado como "algo importante" para el 42,9\% de los usuarios, pero llega a ser "muy importante" para una cifra más reducida de personas: el 34,8 $\%$. Es por ello por lo que, aunque es una finalidad bastante buscada por la audiencia, no se posiciona entre las más destacadas.

El 31,4 \% de los participantes aseguran que acceden al contenido audiovisual online porque les gusta ver contenidos creados por gente como ellos, lo cual valoran como "algo importante". Solo para el 29,3 \% este motivo es considerado como "muy importante".

Otros de los sentidos de utilización más mencionados es el interés que despiertan los contenidos online frente a los de la televisión. El 29,1\% de los encuestados considera que es una razón "muy importante" para elegir ver los vídeos en Internet y el 37,2 \% lo sitúa como "algo importante".

Los usuarios también tienen otros motivos basados en las características que ofrece el consumo audiovisual online. La visualización de vídeos de humor es valorada como "algo importante" por el $40,0 \%$ de los encuestados, mientras que el $32,6 \%$ le aporta la misma relevancia al hecho de que se traten de vídeos creados por youtubers e influencers. Finalmente, el $34,6 \%$ asegura que la corta duración de los vídeos es "algo importante" para ellos.

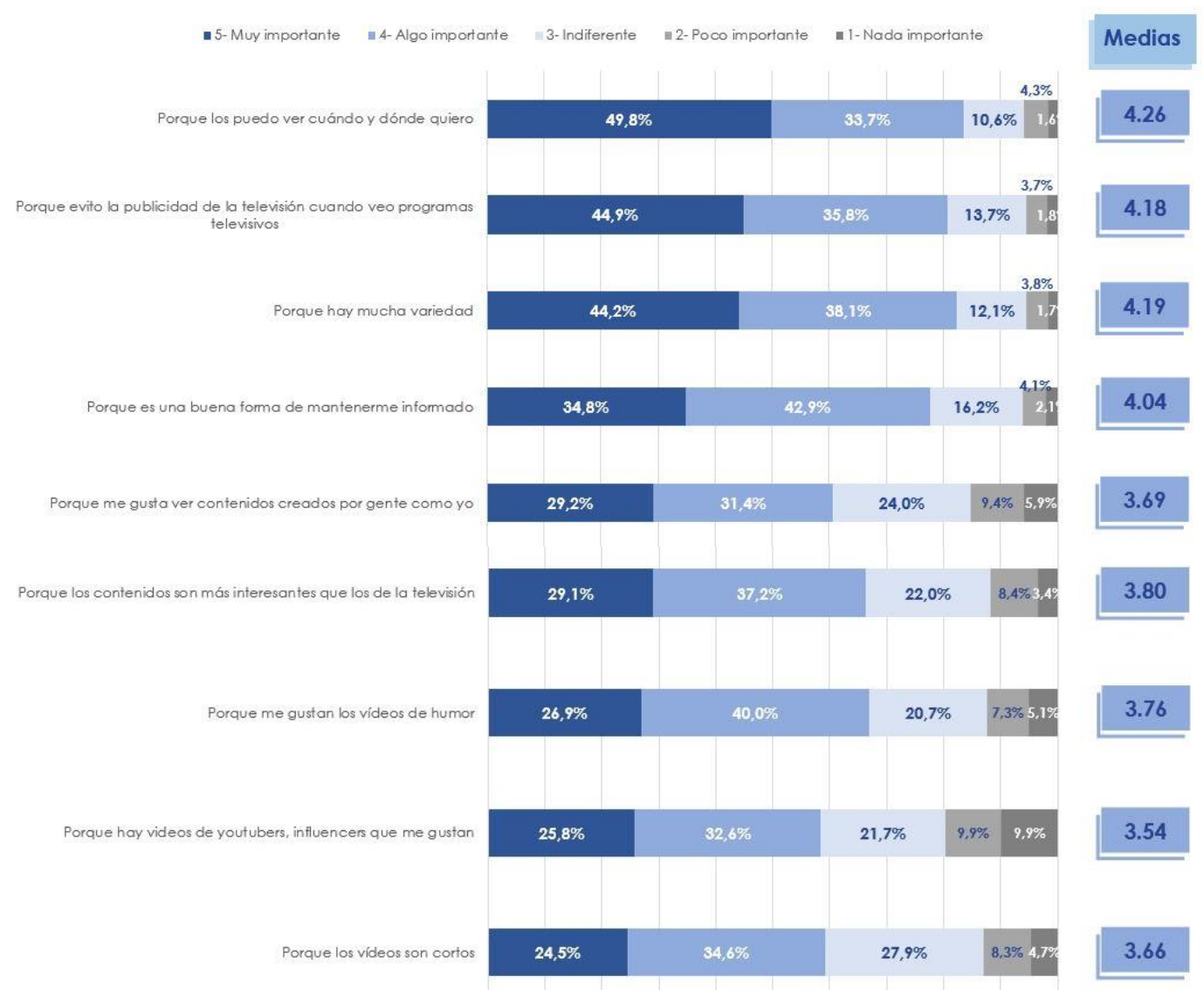

Figura 10. Motivaciones para ver contenido audiovisual online

Fuente: elaboración propia.

Tal y como se podría prever en base a los resultados obtenidos a lo largo del estudio, volvemos a encontrar diferencias relevantes entre los distintos segmentos. Los usuarios jóvenes, sobre 
todo de entre 17 y 24 años, son los que mejor valoran los contenidos audiovisuales online y peor valoran la televisión.

Una de las principales razones para consumir contenidos audiovisuales online es por la posibilidad de evitar la publicidad. Pero también los prefieren frente a televisión porque consideran que su contenido se adapta más y mejor a sus perfiles, puesto que está creado por gente similar a ellos. Además, señalan que el contenido televisivo es de baja calidad y los programas son muy antiguos, ya que los tutoriales, gameplays o vídeos de humor solo están presentes en Internet.

\section{DISCUSIÓN Y CONCLUSIONES}

Tras este análisis concluimos que, aunque la Generación Z comparte hábitos de consumo con las generaciones más mayores, existen determinadas diferencias tanto en sus preferencias de contenido como en la forma de consumirlo. A modo general, podemos determinar que los jóvenes de entre 17 y 24 años son los que mejor valoran los contenidos audiovisuales online.

En primer lugar, respecto a la frecuencia de consumo de contenido audiovisual, destaca el hecho de que este grupo de edad es el que menos visualiza el programa de preferencia por la mayoría de las franjas de edad: los realities shows. Sin embargo, la Generación Z es la mayor consumidora de series de entre todas las encuestadas, sobre todo las mujeres pertenecientes a ella. En concreto, suelen acceder a ellas, al igual que a las películas y las series, a través de cadenas de pago como HBO o Netflix.

Los consumidores audiovisuales tradicionales suelen ver exclusivamente los contenidos en directo y en abierto. Frente a esto, el grupo de audiencia más joven, aunque también consume contenidos televisivos en directo, visualiza significativamente más los propios programas televisivos en diferido y a través de suscripciones de pago, puesto que suele estar más suscrita a plataformas de pago audiovisuales tales como Netflix y HBO. De los consumidores tradicionales, sin embargo, solo un $40 \%$ está suscritos a estas plataformas.

En segundo lugar, tras analizar el soporte utilizado para ver contenido audiovisual, observamos lo siguiente: aunque la mayoría de los consumidores audiovisuales de mayor edad ven los contenidos televisivos en la propia televisión, los nuevos consumidores destacan por su poca utilización de la misma y su diversificación en dispositivos. Estos últimos utilizan mucho más otros soportes como la tablet, el ordenador o el smartphone, que es el dispositivo más utilizado con diferencia.

Existen también diferencias significativas en cuanto a las plataformas online elegidas para ver programas de televisión. Podemos afirmar que los jóvenes de la Generación Z consumen contenido en la red de diversas formas, puesto que los encuestados afirman acceder a varias de las plataformas que les planteamos. Destacan las descargas de Internet, las plataformas de pago como Netflix y también las redes sociales. En cuanto a los programas que suelen ver, llama la atención que no acceden a los informativos a través de ninguna plataforma online, pero obtienen datos muy altos en los deportes, que consumen a través de diversos servicios de pago y también en streaming.

Como podíamos intuir, la Generación Z (así como los usuarios de hasta 35 años) consume muchos más vídeos online que sus antecesores. Sin embargo, la mayoría de los encuestados aseguran que no tienen predilección por las plataformas online frente a la televisión. Este grupo también es el que sube y comparte vídeos por Internet con más frecuencia. 
Finalmente, nos parece muy interesante el hecho de que los nuevos consumidores online valoren de forma negativa la mayoría de los aspectos de la televisión. Consideran que su contenido es de baja calidad y que no cuenta con programas para la audiencia joven, puesto que los que componen su programación son muy antiguos. Puede que este sea el principal cambio de las nuevas audiencias, puesto que los usuarios jóvenes consumen más los medios online por incluir contenidos realizados por personas parecidas a ellos mismos, tales como los tutoriales y los gameplays.

A su vez, también son los usuarios de 17 a 24 años los que mejor valoran los contenidos audiovisuales de Internet. A modo general, los consumidores acuden al contenido audiovisual online por su instantaneidad, la ausencia de publicidad y por aspectos relacionados con el contenido intrínseco de los mismos.

En conclusión, vemos que el consumo audiovisual de la Generación Z no solo está suponiendo un cambio respecto a la tecnología o los soportes utilizados, sino también respecto al contenido al que acceden. ¿Pero cuáles de estos cambios serán asumidos por las siguientes generaciones y cuáles de ellos son solamente algo pasajero?

Mientras que los dispositivos móviles parece que, definitivamente, han llegado para quedarse, ¿sucede lo mismo, por ejemplo, con las plataformas de pago como Netflix? ¿Pueden estas perder preferencia por no ofrecer el mismo nivel de inmediatez o de cercanía que, por ejemplo, ofrece un youtuber o un instagramer mediante su canal o perfil?

Aunque los datos de nuestro estudio son lo más recientes posible, debemos ser conscientes de que los hábitos de consumo de esta generación se encuentran en constante evolución. Como afirma Cerezo (2016), "no hay que olvidar que todos estos análisis se realizan sobre cambios que se están produciendo en tiempo real" (p. 108).

Y para darnos cuenta de ello, no hace falta más que ver cómo se trasladan de unas redes sociales a otras (por ejemplo, con las últimas migraciones de Facebook a Instagram o de Youtube a Twitch) y cómo varían incluso el tipo de uso de cada una de ellas. Es el caso de Snapchat, una red social que los jóvenes españoles han dejado de utilizar para compartir contenido (que era su principal función) y ahora la utilizan solamente por sus filtros: entran a Snapchat, se fotografían con dichos filtros, exportan la imagen y la publican en Instagram.

En consecuencia, consideramos que el estudio del consumo audiovisual de la Generación Z debe ser continuamente actualizado porque, aunque se trata de la generación más analizada, se trata también de la que más posibilidades tiene de evolucionar y sorprendernos.

\section{Referencias}

Aguilar R., M. (2017). La generación Z: Sus hábitos de consumo de información y las redes sociales (Trabajo fin de grado). Universidad de Sevilla, Sevilla, España.

Asociación para la Investigación de Medios de Comunicación (2020). Navegantes en la Red. 22a Encuesta AIMC a usuarios de Internet. Recuperado de https://bit.ly/32zM9bn.

Álvarez R., E., Heredia P., H., \& Romero O., M. F. (2019). La generación Z y las redes sociales. Una visión desde los adolescentes en España. Revista Espacios, 40(20), 1-13. Recuperado de https://bit.ly/2RrFadV.

Aranda, D., Roca, M., \& Sánchez N., J. (2013). Televisión e internet. El significado de uso de la red en el consumo audiovisual de los adolescentes. Quaderns Del CAC, XVI (39), 15-23. 
Barlovento Comunicación. (2019). Consumo de internet vs. televisión. Recuperado de https://bit.ly/2Bh9inz.

Barlovento Comunicación. (2020). Consumo de internet vs. televisión. Recuperado de https://bit.ly/2TTLTiq.

Casanova, J. (2011). Convergencia entre televisión e internet y los modelos de consumo audiovisual. En I. Bort, S. García y M. Martín (Presidencia), Nuevas Tendencias e Hibridaciones De Los Discursos Audiovisuales En La Cultura Digital Contemporánea. Simposio llevado a cabo en el IV Congreso Internacional sobre análisis fílmico, Castellón, España.

Cerezo, P. (2016). La generación Z y la información. Revista De Estudios De Juventud, (114), 95-109.

Espiritusanto N., O. (2016). Generación Z: Móviles, redes y contenido generado por el usuario. Revista De Estudios De Juventud, (114), 111-126.

Francisco-Lens, N., \& Rodríguez-Vázquez, A. I. (2020). La innovación de la televisión pública europea en la oferta audiovisual digital: Nuevas plataformas para la generación Z. RAE-IC Revista De La Asociación Española De Investigación De La Comunicación, 7(13), 185-212. doi:10.24137/raeic.7.13.9

Góngora D., G., \& Lavilla M., D. (2020). Los nuevos hábitos de consumo audiovisual presentes en la era del usuario interactivo. En J. C. Figuereo (Ed.), Estudios multidisciplinarios en comunicación audiovisual, interactividad y marca en la red (pp. 79-95). Sevilla, España: Egregius.

Hernández P., J. F., \& Martínez D., M. Á. (2017). Nuevos modelos de consumo audiovisual: Los efectos del binge-watching sobre los jóvenes universitarios. AdComunica. Revista Científica De Estrategias, Tendencias e Innovación En Comunicación, (13), 201-221. doi:10.6035/332

IAB Spain. (2019). Estudio de TV conectada. Recuperado de https://bit.ly/360II6r.

Instituto Nacional de Estadística. (2019). Encuesta sobre equipamiento y uso de tecnologías de información y comunicación en los hogares 2019. Recuperado de https://bit.ly/3iERPGG.

Jenkins, H. (2008). Convergence culture: La cultura de la convergencia de los medios de comunicación. Barcelona, España: Paidós.

Mouret, S. G. (2016). La generación Z desde la generación Z. Revista De Estudios De Juventud, (114), 157170.

Ortega, F., González I., B., \& Pérez P., M. E. (2015). Audiencias en revolución, usos y consumos de las aplicaciones de los medios de comunicación en tabletas y teléfonos inteligentes. Revista Latina De Comunicación Social, (70), 627-651. doi:10.4185/RLCS-2015-1063

Ortega C., I., Soto S. A., I., \& Cerdán C., C. (2016). Generación Z. El último salto generacional. Recuperado de https://bit.ly/300kzmN.

Ozkan, M., \& Solmaz, B. I. (2015). Mobile addiction of Generation Z and its effects on their social lifes: (An application among university students in the 18-23 age group). Procedia - Social and Behavioral Sciences, 205, 92-98. doi:10.1016/j.sbspro.2015.09.027

Roel V., M. (2019). Aproximación al estudio del consumo televisivo en el ecosistema audiovisual digital español: De la audiencia audimétrica a la audiencia poliédrica. Estudios Sobre El Mensaje Periodístico, (25), 477-492. Recuperado de https://bit.ly/32CMZ7j.

Ruiz S. R., J. A., Cáceres Z., M. D., \& Brändle, G. (2010). ¿Se están modificando los hábitos de consumo audiovisual? El consumo pasivo de televisión en la era de la interactividad. En E. Said (Ed.), Diálogos y desafíos euro-latinoamericanos: ensayos sobre cooperación derecho, educación y comunicación (pp. 211-227). Barranquilla, Colombia: Ediciones Uninorte.

The Center for Generational Kinetics. (2018). The state of gen Z. Recuperado de https://bit.ly/2TKIpil. 
Trifecta Research. (2015). Generation Z media consumption habits. True digital natives. Recuperado de https://bit.ly/3gqAKzz.

Vázquez A., A. (2017). ¿Hacia dónde vamos?: La sociedad enredada. Badajoz, España: AnthropiQa. Recuperado de https://bit.ly/35AITOR.

\section{Notas}

${ }^{1}$ La presente publicación pertenece a una investigación financiada. Este artículo es producto del proyecto de investigación titulado "DE LA CULTURA DE MASAS A LAS REDES SOCIALES: CONVERGENCIA DE MEDIOS EN LA SOCIEDAD DIGITAL" (CSO2016-74980-C2-1-R), financiado por el Programa Estatal de Investigación, Desarrollo e Innovación Orientada a los Retos de la Sociedad, en el marco del Plan Estatal de Investigación Científica y Técnica y de Innovación 20132016. Además, cuenta con el apoyo de un contrato predoctoral del Programa FPU (Formación del Profesorado Universitario) del Ministerio de Ciencia, Innovación y Universidades, con referencia FPU18/00565. 